\title{
The New Laser assisted Wide Angle Tomographic Atom Probe
}

\author{
L. Renaud, ${ }^{1}$ P. Monsallut, ${ }^{1}$ Ph. Benard, ${ }^{1}$ Ph. Saliot, ${ }^{1}$ G. Da Costa, ${ }^{2}$ F. Vurpillot ${ }^{2}$ and B. Deconihout ${ }^{2}$
}

${ }^{1}$ CAMECA, 103 Bd. St-Denis, 92403, Courbevoie cedex, France.

${ }^{2}$ GPM, UMR 6634 CNRS, Institut des matériaux de Rouen, Université de Rouen, 7601 Saint Etienne du Rouvray Cedex, France

The three dimensional Atom Probe (3D-AP) technique can be considered as the only technique which allows 3D imaging and chemical composition measurements capabilities at the atomic (Figure 1). Since its early developments, 3D Atom Probe has provided major contributions in materials science. However, today most of the 3D-Atom Probe instruments suffer from two mains limitations:

- A limited field of view : making difficult to detect small objects of interest (e.g. : clusters) if their concentrations are too small.

- Applicable only to conductive materials.

CAMECA in collaboration with the Atom Probe group from Rouen University (France) has developed a new 3D atom probe : the Laser assisted Wide Angle Tomographic Atom Probe (LaWATAP) to overcome traditional drawbacks of a conventional 3D atom probe. The new features of the La-WATAP are :

- Larger field of view: the field of view has been increased by approximately 5 times. This allows collection of more evaporated atoms over a wider solid angle. It results in the ability to probe a much larger volume of the sample, therefore improving statistical accuracy of compositional measurements. This represents significant acquisition time savings over traditional 3D Atom Probe. Furthermore, the improvement of the field of view has been achieved maintaining high accuracy in composition measurement plus high mass resolution (Figure 2). This is a result of the new position sensitive detector (the advanced delay line detector or ADLD), specially designed to accommodate the high data rate being inherent in wide angle configuration, combined with a laser assisted evaporation system.

- Applicability to non- and semi-conductor materials: the La-WATAP ensures a highly localized atom evaporation at the tip apex, while conventional High Voltage pulses cannot be efficiently propagated along a tip of non conductive materials inhibiting the field evaporation process of single atoms. Thus, the laser evaporation technique is well suited for the analysis of semiconductor materials (Figure 3), thus enlarging the application field of the TAP technique.

The goal of this presentation will be to introduce the main features of the design of the new CAMECA atom probe. Its analytical performances will be demonstrated by application examples selected in metallurgical and semi-conductive materials. 


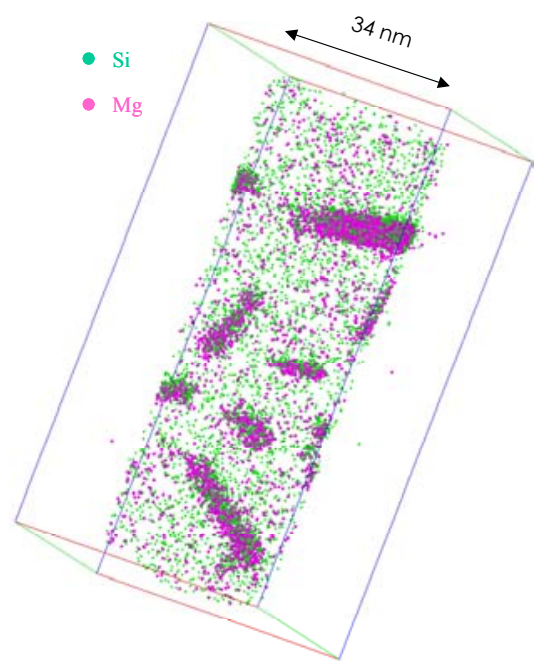

Figure 1 : Precipitation of $\mathrm{Mg} / \mathrm{Si}$ enriched particles in an AlMgSi alloy.

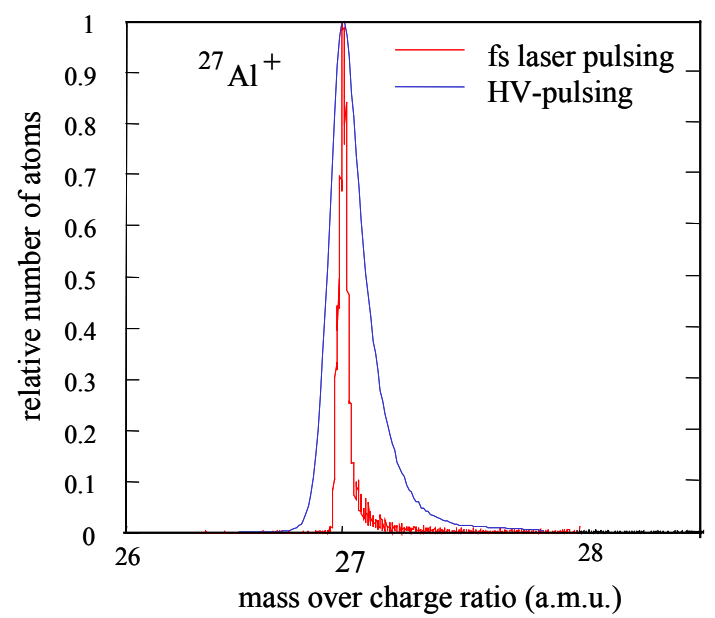

Figure 2 : Mass spectra of an aluminum alloy obtained with HV pulsing (blue curve) and laser pulsing (red curve).
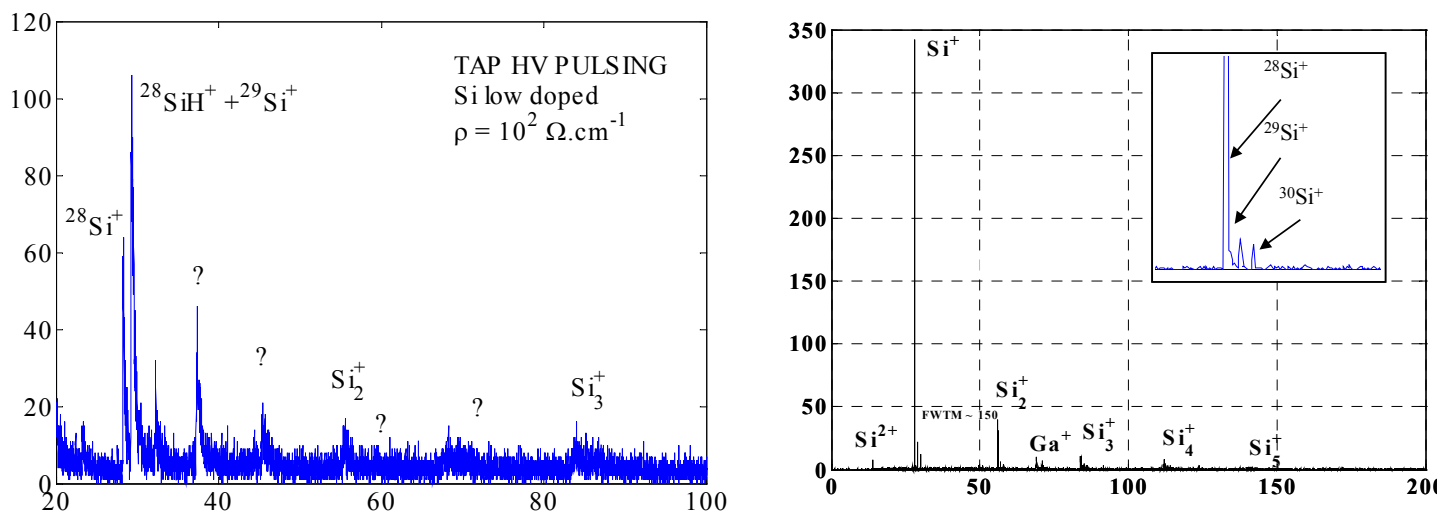

Figure 3 : Mass spectra of a silicon low-doped sample obtained with HV pulsing (left spectrum) and laser pulsing (right spectrum). 